\title{
Retraction: Higher-Order Nonlocal Effects of a Relativistic Ponderomotive Force in High-Intensity Laser Fields [Phys. Rev. Lett. 112, 035002 (2014)]
}

\author{
Natsumi Iwata and Yasuaki Kishimoto \\ (Received 28 July 2015; published 31 December 2015)
}

DOI: 10.1103/PhysRevLett.115.269902

PACS numbers: 52.35.Mw, 45.20.Jj, 52.20.Dq, 52.38.-r, 99.10.Ln

This Letter which was published on 23 January 2014 contains a mathematical derivation for the single particle orbit which was previously published [1] and presented without attribution. Though the objectives and the primary conclusions of this Letter are original, the earlier paper should have been cited and appropriately credited for being a primary source for this work. In addition, the Letter lacks sufficient argumentation for the electrostatic potential on the generalized equation, which is necessary in order for the objectives to be justified clearly. In light of this failure to cite our previous work and of the incomplete organization, the authors retract this Letter and apologize to the readers and editors of Plasma and Fusion Research and Physical Review Letters.

[1] N. Iwata and Y. Kishimoto, Relativistic ponderomotive force including higher order nonlocal effects in high intensity laser fields, Plasma Fusion Res. 8, 1201094 (2013). 